\title{
Cortical Area V4 and Its Role in the Perception of Color
}

\author{
C. A. Heywood, ${ }^{1}$ A. Gadotti, ${ }^{2}$ and A. Cowey ${ }^{1}$ \\ 'Department of Experimental Psychology, Oxford University, Oxford OX1 3UD, United Kingdom and 2Laboratorio de \\ Neurobiologia, Universidade de Brasilia, 70910 Brasilia Df, Brazil
}

\begin{abstract}
The color and lightness vision of three monkeys with bilateral removal of cortical area V4 and three unoperated controls were tested by measuring their ability to discriminate between two rows of colored or gray stimuli. In one row, the stimuli were ordered in terms of either chromaticity or luminance, whereas in the other row they were disordered. Their ability to select the odd-one-out in an array of colors or grays and to select the colored patch from an array of achromatic grays was also assessed. Unlike an achromatopsic patient tested previously in an identical fashion, monkeys with V4 lesions performed indistinguishably from controls in the oddity test. The animals lacking V4 were slightly impaired at discriminating between ordered and disordered arrays of colors or grays, but the color impairment was no more severe than the impairment with grays. These deficits were readily accounted for in terms of the conspicuous deficits in pattern discrimination apparent in a nine-choice pattern oddity task. The results do not support the view that cortical area V4 in the monkey is the homolog of the cortical "color center" in humans, located in the lingual and fusiform gyri and damage to which leads to the clinical syndrome of cerebral achromatopsia, unless it is the additional damage to underlying white matter that leads to the severe color disorder in patients.
\end{abstract}

Onc conscquence of localized cerebral damage in humans can be the complete loss of color vision, a syndrome termed cerebral achromatopsia (for reviews, see Meadows, 1974; Zeki, 1990). It is a condition that can be sharply distinguished from other acquired color vision impairments such as agnosia (an impairment in the recognition of colors) and color anomia (an impairment in the naming of colors), because only the patient with cerebral achromatopsia describes the visual scene as gray or monochrome. It is also readily demonstrated by the Farnsworth-Munsell 100-Hue Test, which requires the patient to order a number of chromatically graded colored disks on the basis of their chromaticity. The ability of achromatopsic patients to perform this task is characteristically grossly abnormal. On the evidence from positron emission tomography in normal observers (Lueck et al., 1989), magnetic resonance imaging in pa-

Received Feb. 11, 1992; revised May 8, 1992; accepted May 20, 1992

This research was supported by a grant from the Medical Research Council (G971/397/B) and by a Human Frontier Science Program grant. We are especially grateful to David Popplewell for his help in programming computer-controlled visual displays. A.G. thanks the CNPQ (Brazil) for a traveling fellowship.

Correspondence should be addressed to Dr. C. A. Heywood, Department of Experimental Psychology, South Parks Road, Oxford OX1 3UD, UK.

Copyright (C) 1992 Society for Neuroscience $0270-6474 / 92 / 124056-10 \$ 05.00 / 0$ tients (IIeywood et al., 1991), and postmortem assessment of achromatopsic patients (Meadows, 1974; Damasio et al., 1980), the lingual and fusiform gyri in the ventral portions of the occipital lobe are considered to be the crucial regions involved in the processing of chromatic information that leads to the conscious experience of hue.

Such evidence has led to the proposed existence of a localized "color center" in primate visual cortex, a region specialized for the processing of chromatic information. This view has been bolstered by the demonstration in nonhuman primates of a multiplicity of visual areas in prestriate cortex and the suggestion that different areas are specialized for the processing of different visual attributes. Thus, the color and wavelength selectivity shown by a large proportion of cells in cortical area V4 suggests that it is an area specialized for color vision. This can be contrasted with the properties of cells in area MT (V5) that are predominantly direction sclective and those in areas V3 and $\mathrm{V} 3 \mathrm{~A}$ where the majority of cells are orientation selective. In none of these areas is wavelength selectivity apparent.

The segregation of pathways dealing with color, motion, and form precedes these areas and is also apparent within area V1 (see Livingstone and Hubel, 1987, for review). The blobs and interblobs in area $\mathrm{V} 1$, revealed by cytochrome oxidase staining, receive projections from $P \beta$ retinal ganglion cells (Perry et al., 1984) via the parvocellular layers of the dorsal dLGN and constitute the chromatically opponent $P$ channel. About half of the cells in the blobs are wavelength selective, but not orientation selective, whereas cells in the interblobs are predominantly selective for orientation. Area V2 has a characteristic pattern following staining for cytochrome oxidase that reveals thick and thin stripes separated by interstripes. Cells in the interstripes receive projections from interblobs and are orientation selective but not wavelength sclective. The blobs project to the thin stripes where the majority of cells are not orientation selective but wavelength selectivity is present in some $50 \%$ of them. The thin stripes and interstripes relay their information to V4. Thus, the wavelength and orientation selectivity of cells that constitute the $P$ channel, which relays information to area V4, suggest that this channel is concerned with the processing of color and form. It is hardly surprising that the representation of the $P$ channel in area V4 is seen to provide an explanation for cortical achromatopsia in humans when brain damage includes a region assumed to be homologous and functionally analogous to V4 in monkeys.

The second channel (the $\mathrm{M}$ channel) arises from the $\mathrm{P} \alpha$ retinal ganglion cells (Perry et al., 1984) that project to the magnocellular cells of dLGN, which in turn innervate layer $4 \mathrm{~B}$ of cortical area V1. From there, information is relayed to area MT (V5) 
and areas V3 and V3A via the cells in the thick stripes of V2. The orientation sensitivity and absence of wavelength selectivity that are apparent in the cells of the thick stripes in V2, along with the properties of cells in areas V3, V3A, and MT, are consistent with the proposed role of the latter areas in the processing of form and motion, respectively. There are thus two pathways for the processing of form, associated with the $\mathrm{P}$ and $M$ channels, respectively, although this should not be allowed to obscure increasing evidence that the two channels are not completely segregated. For example, there are $\mathrm{P}$ channel inputs to area MT (Maunsell et al., 1990).

While cells in V4, and their connectivity, show just the properties required for the computational procedures proposed for the construction of colors (Land, 1974), it is not yet established whether the "color center" in humans is the homolog of cortical area V4 in the nonhuman primate. What is required, in the absence of convincing physiological or neuroanatomical markers in the human brain, is an assessment of color and form vision in monkeys following ablation of area V4. An earlier study (Heywood and Cowey, 1987) demonstrated a mild impairment in hue discrimination following bilateral V4 lesions with a spared ability to discriminate achromatic grays. In addition, however, the monkeys showed a severe impairment in the discrimination of form. Similar results have been reported following partial lesions of V4 when color discrimination was tested in the corresponding part of the visual field (Schiller and Logothetis, 1990; Schiller and Lee, 1991). Achromatopsia is a gross impairment in the discrimination of color (when tested with the Farnsworth-Munsell 100-Hue Test) and is commonly associated with reduced acuity and mildly impaired form discrimination, although the recognition of specific forms, such as faces, may be severely impaired. However, form vision can be totally intact, and cerebral achromatopsia is therefore unlike the pattern of deficits that follow V4 removal in the monkey. However, assessment of discrimination was previously made in achromatopsic patients (Heywood et al., 1987, 1991) and in monkeys with V4 lesions (Heywood and Cowey, 1987) by requiring the subject to select the odd-one-out in three Munsell color swatches. The results for patients and monkeys were similar. Performance was almost errorless for the easiest discrimination, for example, red versus orange, but was impaired with closely similar hues. Nevertheless, the same achromatopsic patients showed a profound disturbance in the ordering, identification, and classification of hues and complained about their poor color vision.

One possible explanation of the relatively good performance on discrimination tasks is that using Munsell swatches in threechoice oddity underestimates the extent of the impairment in monkeys with V4 lesions, just as it does in achromatopsic subjects. Munsell papers are designed to be isoluminant for the fovea of a hypothetical average young observer and may well depart from isoluminance for papers that are chromatically widely separated and when viewed by an older or nonaverage observer or by monkeys. Selecting the odd color in a group of three colors might be achieved by discriminating on the basis of brightness. We therefore examined the ability of monkeys before and after V4 lesions to perform tasks of chromatic and achromatic ordering along with color discriminations in a variety of oddity tasks where brightness is irrelevant. Identical tasks were previously carried out by an achromatopsic patient, enabling comparisons to be made. Finally, form discrimination was assessed in an oddity test.

\section{Materials and Methods}

\section{Subjects}

Six previously untrained male cynomolgus monkeys (Macaca fascicularis) were used. They weighed between 3 and $4 \mathrm{~kg}$ at the start of training. All six were tested on a variety of color and form discriminations. Area V4 was then removed in three animals (operated group), and they were retested along with the unoperated animals (control group).

\section{Surgery}

The monkey was sedated with ketamine hydrochloride (Vetalar, $10 \mathrm{mg}$ / $\mathrm{kg}, \mathrm{i} . \mathrm{m}$.) and then anesthetized with sodium thiopentone (Intraval, i.v.). Deep anesthesia was maintained with supplementary intravenous injections. All surgical procedures were carried out under strict asepsis.

The monkey was placed in a head holder, and following a midline incision of the skin, the temporal muscle on both sides was cut vertically and retracted laterally and ventrally. Using rongeurs, an opening was made in the bone over the prelunate gyrus, which was then exposed by cutting and reflecting the overlying dura matter. With the aid of an operating stereomicroscope and using subpial aspiration, the cortex on the prelunate gyrus was removed from the junction of the lunate and superior temporal sulci dorsomedially to the inferior occipital sulcus ventrolaterally. The ablation was extended a few millimeters into all three sulci. A similar band of cortex was then removed from the lower bank of the ascending limb of the inferior occipital sulcus to a line just beyond the occipitotemporal sulcus on the ventral surface of the brain. The entire band of cortex corresponds to the area of V4 as described by Ungerleider (1985), although the exact borders of the ventral region have never been precisely mapped in macaque monkeys. After any bleeding had ceased, the dura, muscle, fascia, and skin were sutured and the animal was given $0.1 \mathrm{ml}$ of Temgesic to reduce any postoperative discomfort. All animals regained consciousness within $2 \mathrm{hr}$, and recovery was uneventful.

\section{Histology}

At the end of the experiment, each animal was sedated with Vetelar and given a lethal dose of sodium pentobarbitone (Sagatal, i.v.). After clamping the descending aorta, the brain was perfused through the heart with $500 \mathrm{ml}$ of $0.9 \%$ saline followed by $10 \%$ formalin in $0.9 \%$ saline, all at room temperature. With the head in a stereotaxic frame, the overlying skull was removed and the brain was blocked in the stereotaxic frontal plane. The brain was then removed, photographed, and infiltrated with a solution of $30 \%$ sucrose in formal saline for 1 week. Frozen sections were then cut at $25 \mu \mathrm{m}$, and every tenth section was stained with thionine for Nissl substance. An additional 1 in 20 series was stained for fibers according to the method of Gallyas (1979).

The results of the histological examination are shown in Figure 1. In both hemispheres of all three animals, the intended area had been removed. The prelunate gyrus was missing, and as a result, the lunate and superior temporal sulci had shifted toward each other, minimizing the representation of the rostrocaudal extent of the dorsolateral ablation. Ventrally, the ablation reached or crossed the occipitotemporal sulcus hilaterally in all three monkeys but was most extensive in V4-1, where it clearly involved area TEO, adjacent to V4. In every monkey, the rostral bank of the lunate sulcus and both banks of the tip of the inferior occipital sulcus were missing. There was no detectable damage to the dorsal lateral geniculate nucleus or any other underlying structure, apart from the lateral and inferior pulvinar, where there was thinning of neurons and evidence of gliosis, as would be expected after damage to extrastriate visual cortex. These retrograde changes were essentially identical to those reported earlier after similar lesions (Heywood and Cowey, 1987).

\section{Apparatus}

Visual stimuli were presented on a 20 inch Mitsubishi color monitor with long-persistence phosphors. The color gamut of the three primary phosphors is shown in Figure 2, using conventional CIE (Commission International a'Eclairage) coordinates. The screen was controlled by a Pluto II graphics device (Electronic Graphics Ltd.) providing a screen resolution of 768 pixels horizontally by 576 pixels vertically. Each of the red, blue, and green guns had an 8-bit resolution, which allowed the presentation of a choice of 256 simultaneous colors from a palette of $256^{3}$. For achromatic stimuli, the choice was restricted to 256 gray levels.

The monkey sat in a wheeled transport cage placed in front of the 

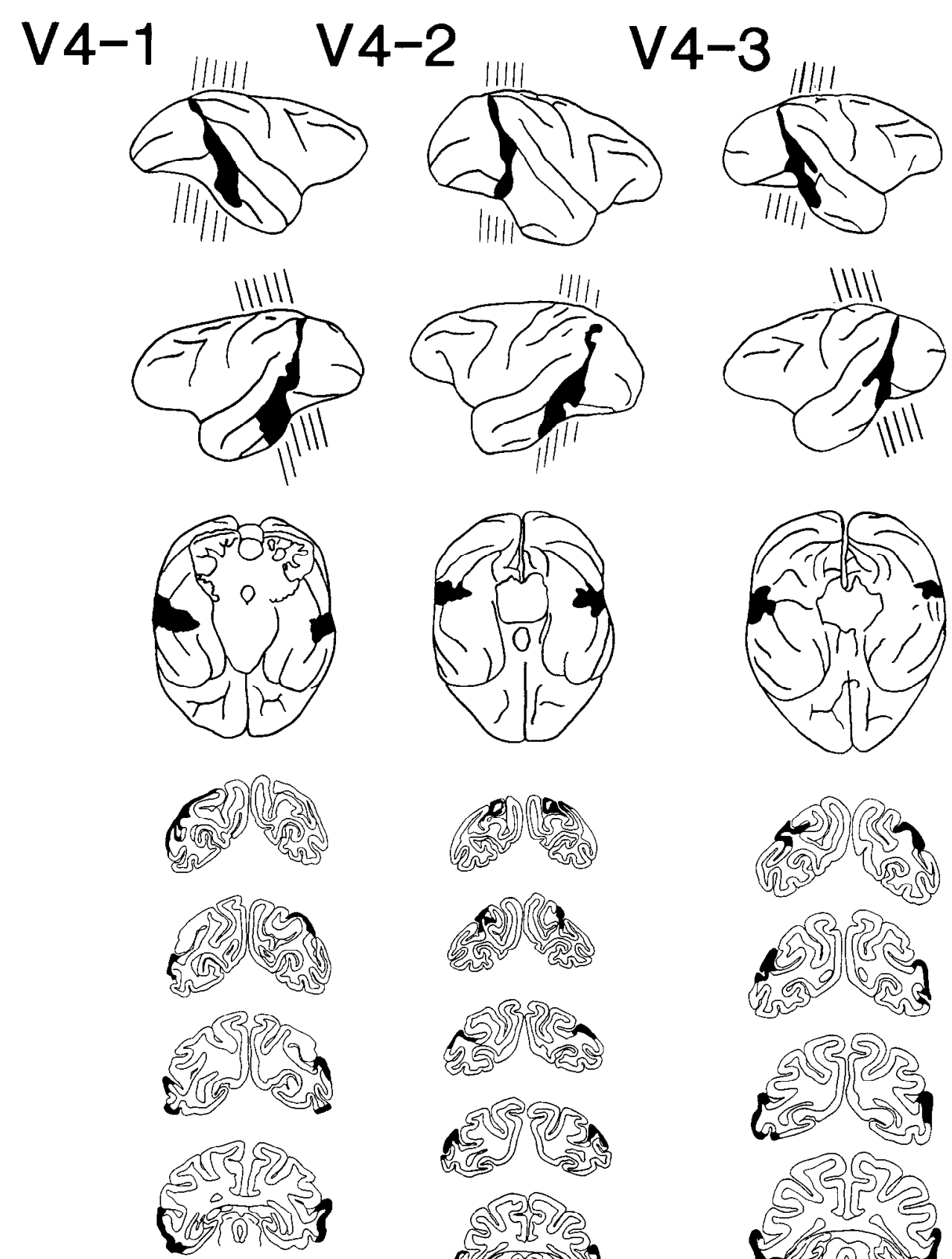

Figure 1. Reconstruction of the cortical ablations to area V4 in three monkeys. In each column, the right, left, and ventral views of the brain are shown, followed by drawings of frontal sections, at the levels indicated alongside each of the lateral views of the hemispheres. The right hemisphere is shown on the right of each outline drawing. Missing tissue is shown in solid black.
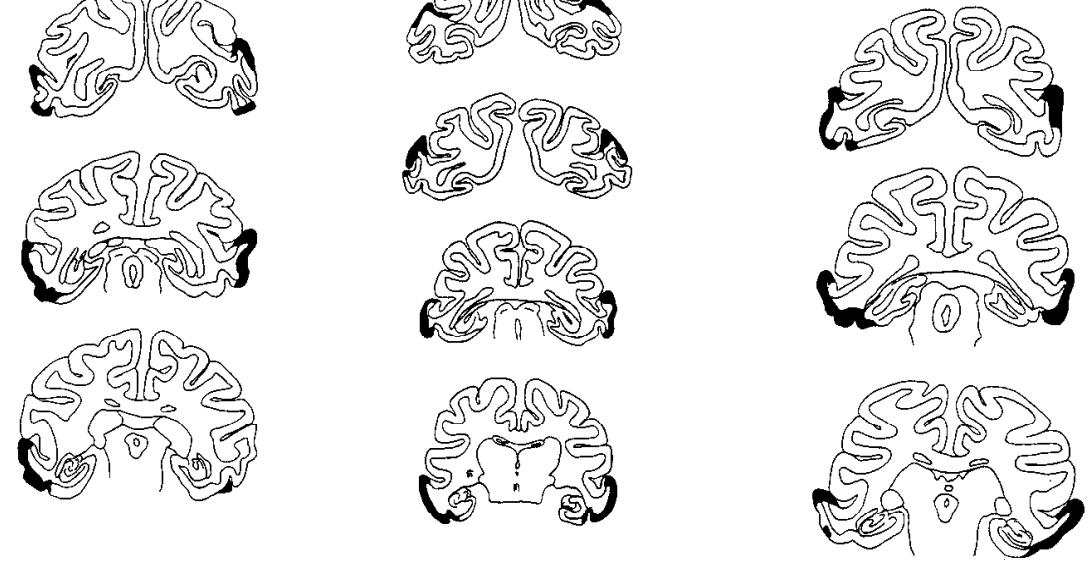

monitor. He could respond to visual stimuli by reaching through the bars of the cage and touching a glass window placed against the front of the monitor. A grid of infrared beams traversed the surface of the window at $1 \mathrm{~cm}$ intervals. Each beam was detected by a photocell that was tuned to the high-frequency infrared signal. The response was recorded by the interruption of one or more of these beams. The touchsensitive screen and graphics controller were interfaced with an IBM microcomputer. Appropriate responses were rewarded by delivering a peanut to a food well centrally positioned below the screen. Ambient illumination in the testing cubicle was provided by light from the monitor at a hackoround screen luminance of $16.0 \mathrm{Cd}^{\prime} \mathrm{m}^{2}$. All fuminance measurements were made with a Minolta LS-110 digital luminance meter calibrated for human CIE photopic spectral sensitivity. Animals were observed by the experimenter from an adjacent room via video camera and monitor.

Visual discriminative stimuli

For chromatic and achromatic discriminations, stimuli were composed of patches of isoluminant colors or achromatic grays. Measurements were made of the brightness of each of the red, green, and blue guns for each of the 256 input voltages. Series of isoluminant chromatic stimuli were selected by an appropriate mixture of the three guns. The position of each stimulus was plotted on the CIE chromaticity diagram in the following manner. Tristimulus values were calculated where

$$
X=\mathrm{LR}(x r / y r)+\mathrm{LG}(x g / y g)+\mathrm{LB}(x b / y b),
$$


and

$$
Y=\mathrm{LR}+\mathrm{LG}+\mathrm{LB},
$$

$$
Z=\mathrm{LR}(z r / y r)+\mathrm{LG}(z g / y g)+\mathrm{LB}(z b / y b)
$$

Each stimulus is defined by a combination of $\mathrm{LR} \mathrm{Cd} / \mathrm{m}^{2}$ of red, $\mathrm{LG}$ $\mathrm{Cd} / \mathrm{m}^{2}$ of green, and $\mathrm{LB} \mathrm{Cd} / \mathrm{m}^{2}$ of blue, where $\mathrm{LR}, \mathrm{LG}$, and $\mathrm{LB}$ refer to the luminance of each of the 3 phosphors. Coefficients $x r, y r, z r, x g$, $y g, z g, x b, y b$, and $z b$ refer to the CIE chromaticity coordinates of the red, green, and blue phosphors derived from telespectroradiometry measurements. Chromaticity coordinates for the combination were then calculated in the usual manner, that is, $x=X /(X+Y+Z)$ and $y=$ $Y /(X+Y+Z)$.

Eleven series of colors were used, each ordered in chromaticity. Ten series were composed of five colors in each and were used for tasks of color ordering. The luminance of each series varied between 7.7 and $42.7 \mathrm{Cd} / \mathrm{m}^{2}$. The eleventh series was composed of nine colors in the red/green range created by the addition of the red and green guns in varying proportions while maintaining a luminance of $12.4 \mathrm{Cd} / \mathrm{m}^{2}$ for every stimulus. These colors were used in nine-choice and three-choice color oddity tasks. The position of each series on the standard CIE diagram is indicated in Figure 2. The values we chose were confirmed to be within the range of values for isoluminance derived from flicker photometry in several human subjects, including ourselves.

For achromatic discriminations, 170 gray levels were used, ranging from $0.7 \mathrm{Cd} / \mathrm{m}^{2}$ to $38.03 \mathrm{Cd} / \mathrm{m}^{2}$ with the constraint that no stimulus had a luminance difference of less than $5 \mathrm{Cd} / \mathrm{m}^{2}$ from the background.

Series of achromatic stimuli were composed of five patches of achromatic grays where differences between adjacent grays was of the order of $1 \mathrm{Cd} / \mathrm{m}^{2}$. These stimuli were used for tasks in which the monkeys had to indicate which of two rows of gray stimuli was uniformly ordered. A further range was selected for three-choice and nine-choice oddity discrimination. A series of nine grays was selected, ranging between 2.09 and $18.82 \mathrm{Cd} / \mathrm{m}^{2}$ in steps of approximately $2 \mathrm{Cd} / \mathrm{m}^{2}$.

Twenty-six alphanumeric characters (Hershey characters 3001-3025) were used for the discrimination of form. The characters were drawn with $2.5 \mathrm{~mm}$ line widths and were not more than $2 \mathrm{~cm}$ high by $1 \mathrm{~cm}$ wide.

\section{Procedure}

\section{Pretraining}

The monkeys were trained to touch a $10 \mathrm{~cm}$ white square that appeared against a gray background at the center of the screen. The square appcared with an intertrial interval of $5 \mathrm{sec}$ and remained on the screen until touched. If the monkey touched the square, the screen became blank and a peanut was delivered. Animals received 50 trials per day until they were reliably responding. The square was then replaced by a white alphanumeric character, the letter "A," which appeared from trial to trial at random positions on the screen. The animals rapidly learned to touch this stimulus for a peanut reward. Eight identical characters that differed from the target character were then introduced onto the screen in random positions. For this purpose, the screen was divided into an imaginary $9 \times 9$ grid that defined possible positions. Only one stimulus appeared within each of the vertical columns on the grid, which were separated by $3 \mathrm{~cm}$, and only one stimulus appeared in each row. The required response was to touch the letter " $A$ " while ignoring the irrelevant items. If an incorrect item was touched, an error was recorded and the trial cnded. On all trials, the target remained the same but the character comprising the identical unrewarded stimuli changed in form and color from trial to trial. The spatial position of these items was randomized from trial to trial within the constraints described above. There were 100 trials per day. The intertrial interval for this and subsequent tasks was $5 \mathrm{sec}$. Within $2 \mathrm{~d}$, all six monkeys were making more than $80 \%$ correct responses. For the following $2 \mathrm{~d}$, the target item now changed randomly in color and form but the eight incorrect characters remained unchanged from trial to trial. The colors were selected from an arbitrarily selected palette of 256 colors. Again, within $2 \mathrm{~d}$ performance was better than $80 \%$ correct.

\section{Preoperative testing}

Examples of visual displays for tasks described below are presented in Figure 3.

\section{Form discrimination}

For $10 \mathrm{~d}, 100$ trials a day were presented where the target and the unrewarded stimuli were changed every 10 trials, although the spatial

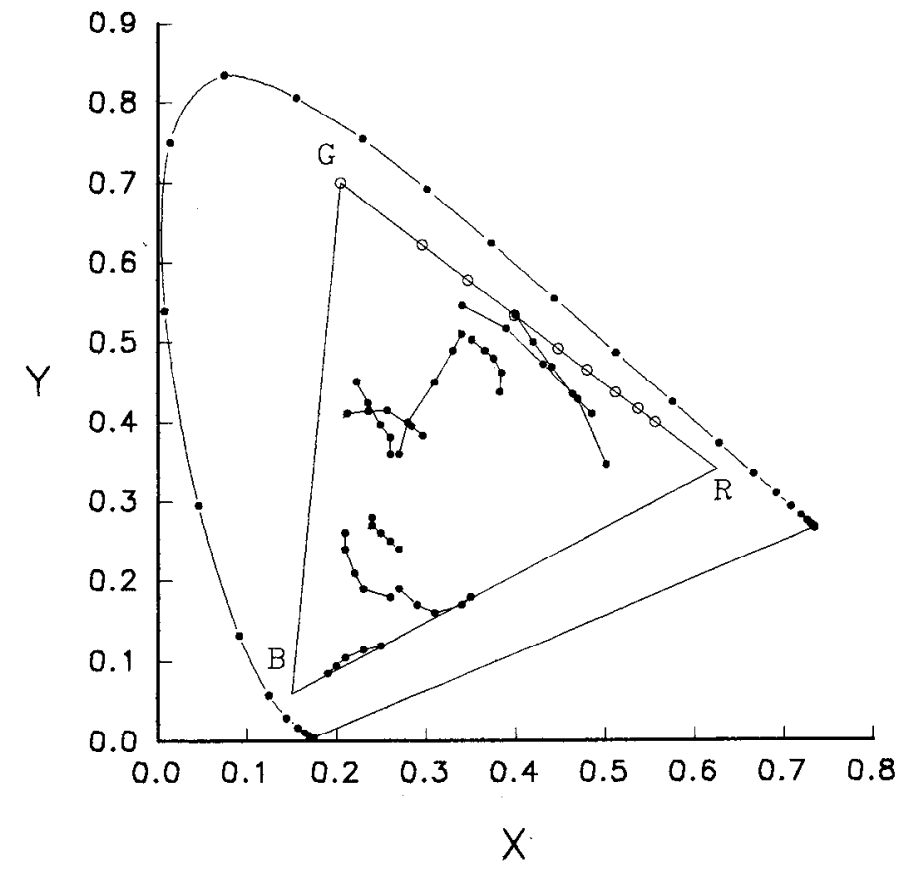

Figure 2. The apices of the triangle $R G B$ show the CIE coordinates of the three phosphors of the color monitor plotted on to the standard CIE diagram. The nine colors indicated by open circles between $G$ and $R$ are the colors constructed from various mixtures of the red and green guns and used in the tasks of ordering, oddity, and two-choice discrimination in the red/green range. The other 10 series of five colors each (solid circles) were used for the perception of color ordering in other parts of color space. Examples of stimuli are shown in Figure 3.

arrangement of the stimuli was randomized from trial to trial; that is, there were 10 trials/problem and for any trial the animal had to touch the stimulus that differed from the other eight. For the final $10 \mathrm{~d}$, the task was identical to that just described except that the color of the target item was the same as the other eight and the oddity discrimination was solely based on differences in form. These tasks yielded preoperative performance measures for the discrimination of form.

\section{Ordering of hues and grays}

In the Farnsworth-Munsell test of color vision, the subject has to arrange a number of colored or gray disks, typically 20 , into an orderly chromatic or gray series. Patients with cerebral achromatopsia sort the colored disks in a manner that is often no different from a random arrangement. We therefore devised an analogous test for monkeys where they had to indicate which of two simultaneously presented rows of stimuli was chromatically or achromatically ordered. Each discrimination was between two horizontal rows of colors or grays that were presented one $10 \mathrm{~cm}$ above the other. Each row consisted of five contiguous $1.5 \mathrm{~cm}$ squares of color or gray. In one row, the colors or grays were in an orderly sequence of chromaticity or luminance and each order could be from left to right or vice versa. In the other row they were jumbled. The task was to select the ordered row by touching it. The spatial position of the rewarded stimulus, that is, above or below, was varied from trial to trial with the constraint that no position was rewarded for more than three consecutive trials. Testing involved a daily session of 100 trials for $5 \mathrm{~d}$ per week. Correct responses were rewarded with a peanut. Incorrect responses were signaled by switching off the screen for $5 \mathrm{sec}$, leaving the animal in darkness. Trials were terminated after the response had been made, when stimuli disappeared from the screen. Stimuli were randomly selected for any particular trial by the random-number generator, seeded with a session number, so that an identical series of 100 trials could be repeated. In every case, the background luminance of the screen was $16.0 \mathrm{Cd} / \mathrm{m}^{2}$. Four tasks were presented.

Task 1 . For any trial, a series of ordered grays was selected from a random starting position in the total set of achromatic discriminanda. The unrewarded stimulus was composed of the same grays jumbled randomly. 


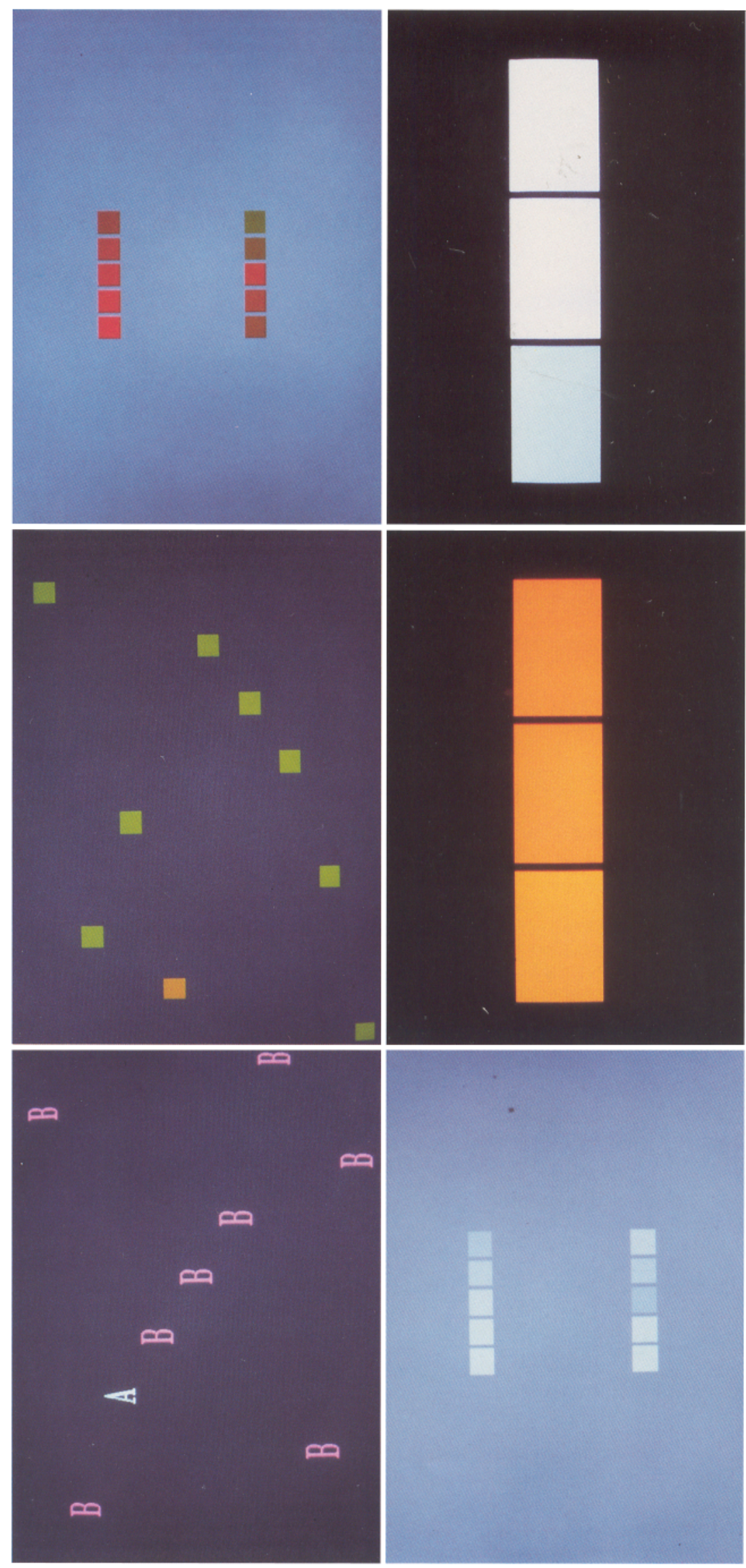

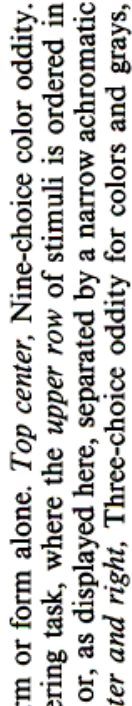

영

들

เ

응 항 융

덩 요잉

을

密势

츄

묵훈

\&ᄋ

캐는

ذं

을

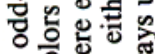

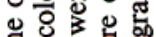

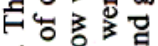

品 댄 ㄴ. 目告为 예. 8 E 을 0 递号. $Z$ w

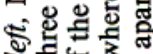
20 $\checkmark$ -

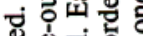

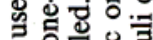
n 讨息 . 0 웅 $\partial \equiv$.

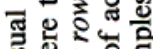
no टे 要 4

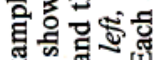

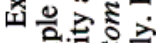
․․ㄹㅇ n 류 질

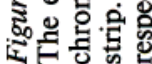


Task 2. As above, but the unrewarded stimulus was a jumbled series of grays also selected from a random starting position in the total set of achromatic discriminanda. Thus, the rewarded stimulus could be an orderly sequence of grays (all light), and the unrewarded stimulus, a jumble of different grays (all dark).

Task 3. The stimuli were as in task 2, but instead of varying between black and white, they spanned the range of red to green. The stimulus set is indicated in Figure 2.

Task 4. The stimuli were the 10 series of isoluminant colors specified in Figure 2, but the rewarded and unrewarded stimuli were selected randomly, and the unrewarded stimulus was again jumbled in a random fashion; that is, the positive stimulus might be an orderly transition from red to green and the negative stimulus a jumbled series from red to yellow.

Each task was presented until $85 \%$ correct performance was achieved in a single session.

\section{Postoperative testing}

Postoperative testing refers to all testing carried out after removing area V4 or to the identical testing carried out for the second time in the three unoperated control monkeys.

\section{Form discrimination}

The monkeys were tested on the discrimination of alphanumeric characters in a manner identical to that described for preoperative testing.

\section{Ordering of hues and grays}

The procedure for testing of the ordering of chromatic and achromatic series was identical to that used preoperatively. Testing continued until animals scored $85 \%$ correct in a single scssion. Subscquently, the monkeys were presented with tasks identical to the first and third ordering tasks, namely, the ordering of achromatic grays and the red/green series. However, now the patches were no longer contiguous but were laterally separated from one another by $5 \mathrm{~mm}$. This alteration mimics the arrangement used with patients in the Farnsworth-Munsell test, where the actual hues can never be contiguous because each is surrounded by a black rim about $5 \mathrm{~mm}$ wide.

\section{Oddity for hues and grays}

Performance for hue and gray discrimination was assessed in both a nine-choice and a three-choice oddity paradigm. In the former, four tasks were presented, each composed of 500 trials.

(1) Nine-choice oddity for colors. The colors were the nine colors specified in Figure 2, varying in chromaticity between red and green. Each monkey received 500 trials where, for any trial, the colors of the sole positive (rewarding) stimulus and the eight identical negative (unrewarding) stimuli were selected randomly from the nine available discriminanda. This resulted in an increasing number of trials for the more difficult discriminations; that is, the most difficult discrimination between adjacent colors in the chromatic series occurred on average nine times more frequently than the easiest discrimination between the most widely separated colors.

(2) Nine-choice oddity for grays. The grays were a set of nine selected from the same stimulus set as had been used for the ordering tasks and consequently differed in luminance by at least $1 \mathrm{Cd} / \mathrm{m}^{2}$. Again, the discriminanda were selected for each trial in the manner described above, with the constraint that the positive and negative discriminanda were always more that two steps apart in the available series of achromatic stimuli.

(3) Nine-choice oddity where the rewarded stimulus was a colored square, randomly selected from trial to trial from the 50 colors used in ordering of hues (task 4, above), embedded in eight gray squares, each of a different luminance that differcd from trial to trial. Brightncss was therefore totally irrelevant.

(4) As above except that the positive stimulus was a red square, which varied in luminance from trial to trial, embedded in eight green squares, each of a different luminance, which also differed from trial to trial.

In an earlier experiment (Heywood and Cowey, 1987), we studied performance on three-choice oddity for real Munsell color and gray cards in macaque monkeys with V4 lesions. We therefore included the same procedure in the present experiment in case the precise stimulus display and response requirements were important.

For the three-choice oddity test, a horizontal row of three rectangular patches $(8 \mathrm{~cm} \times 5 \mathrm{~cm})$ scparatcd by $5 \mathrm{~mm}$ were presented. Two patches were identical, and the third differed in the appropriate dimension of hue or brightness and only appeared at the left or right of the stimulus display. The monkeys were required to touch the odd-one-out. Fifty trials were presented where for each trial the discriminanda were randomly selected from the red/green range used in the ordering of isoluminant colors (Fig. 2). The same procedure was repeated with threechoice oddity for grays, again using a series of nine achromatic stimuli. In each case, 500 trials were presented.

\section{Two-choice hue discrimination}

The discriminanda for two-choice discrimination were the first four greens used in the red/green series (Fig. 2), arbitrarily labeled 1, 2, 3, and 4 . They were identical in size to those used in three-choice oddity. For any discrimination, two stimuli were presented, separated horizontally by $8 \mathrm{~cm}$. All other procedures were identical to those used in the other two-choice discrimination tasks with the exception that animals were tested until a criterion of $90 \%$ correct was achieved in a single session. Three discriminations were presented: $1^{+}$versus $4^{-}, 3^{+}$versus $2^{-}$, and $3^{+}$versus $4^{-}$, in that order.

\section{Results}

Ordering tasks

Performance on tasks of chromatic and achromatic ordering was analyzed by ANOVAs. Saving scores were calculated conventionally as the difference between the errors required to reach $85 \%$ correct performance before and after operation for the V4 group (and the first and second occasion for the control group), divided by their sum and expressed as a percentage, for each of the four tasks. Error scores included criterial trials. ANOVA was carried out on these scores.

All animals readily learned the first task, namely, the discrimination between two achromatic series where the corrcct and incorrect choices comprised an ordered or jumbled array of different grays (errors to criterion, $142 \pm 66$, mean \pm SD). As illustrated in Figure 4, animals with V4 lesions showed substantial negative savings on three of the four ordering tasks and overall were significantly impaired $(F=26.57$; df 1,$4 ; p<0.01)$. Savings were significantly greater for the relearning of the first task $(F=11.56 ;$ df 3,$12 ; p<0.01)$, and this was true for both groups. In addition, and importantly, animals with V4 lesions were no worse at the chromatic than the achromatic discriminations $(F=0.68$; df 3,$12 ; p>0.05)$.

Since saving scores conceal information about the difficulty of tasks in terms of the absolute number of errors committed in reaching criterion, an ANOVA of the number of errors made in reaching criterion, pre- and postoperatively for each task, was carried out. Animals with V4 lesions showed a large increase in the number of errors made in reaching critcrion postopcratively $(F=65.69$; df 1,$4 ; p<0.01)$. Discrimination between ordered and disordered hues, where the hues were selected from a larger set than the red/green range, was of greater difficulty than any of the other three tasks $(F=8.48$; df 3,$12 ; p<0.01)$, and this was true for both groups $(F=1.96$; df 3,$12 ; p>0.05)$. Animals with V4 lesions committed 772 errors $(\mathrm{SD}=233)$ in reaching criterion postoperatively, compared with 129 errors $(\mathrm{SD}=58)$ for the unoperated group at retesting. However, within-subject comparison of performance on ordering of achromatic (task 2) and chromatic, red/green (task 3) stimuli failed to yield significant differences $(t=-0.26$, df $12, p>0.05 ; t=$ 1.09 , df $12, p>0.05$, for the operated and unoperated group, respectively). Operated animals made a mean of $200(\mathrm{SD}=86)$ and $209(\mathrm{SD}=27$ ) errors at retesting for the two chromatic and achromatic tasks, respectively, compared with $74(\mathrm{SD}=61)$ and 


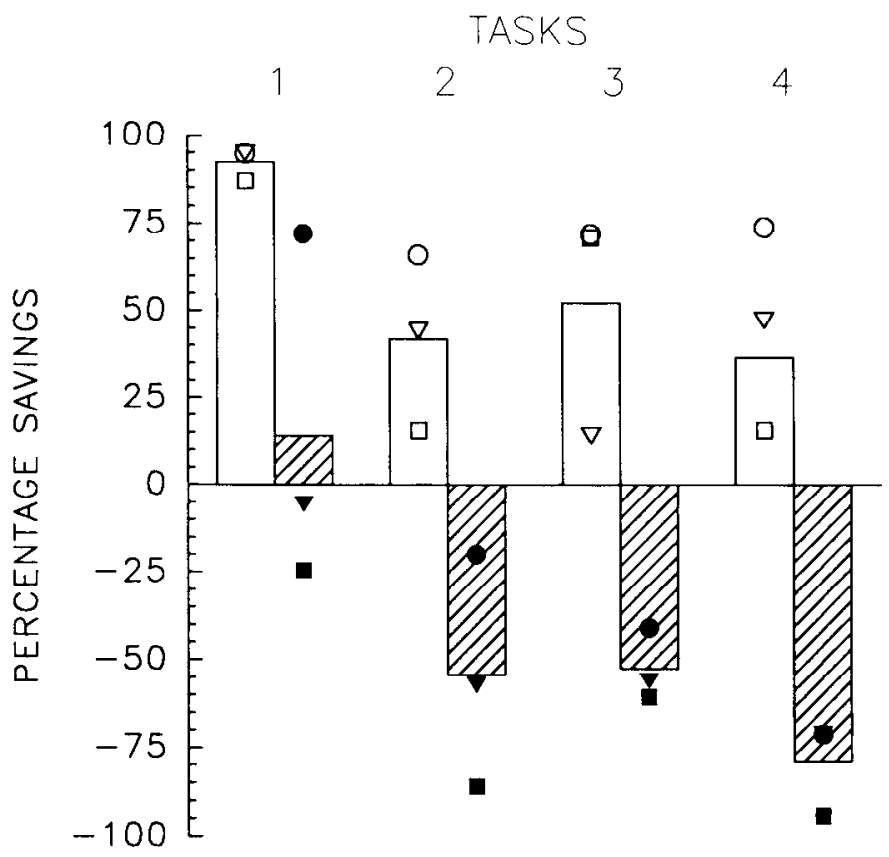

Figure 4. Each pair of bars, from left to right, represents mean percentage saving scores for each of the four ordering tasks, $1-4$. A score of zero indicates that the same number of errors was made on retesting. Positive savings indicate that fewer errors were made the second time. Negative savings indicate that more errors were made the second time. Scores for the unoperated control group are shown as open bars and for animals with V4 lesions as hatched bars. Scores for individual animals are presented as solid and open symbols for the unoperated and operated groups, respectively. In the case of the V4 group, the individual animals are V4-1, squares; V4-2, circles; V4-3, triangles.

$30(\mathrm{SD}=15)$ errors for the unoperated control group. There was therefore no evidence of a selective deficit in the ordering of colored, as opposed to gray, stimuli.

Separating the patches so that adjacent patches were no longer contiguous did not alter the results. For the achromatic ordering task, unoperated and operated animals made $35(\mathrm{SD}=24)$ and $41(\mathrm{SD}=42)$ errors, respectively $(t=-0.03$; df $4 ; p>0.05)$. When chromatic patches were separated, all animals found the task substantially more difficult and the criterion of $85 \%$ correct was reached by only one animal while the remaining animals failcd to scorc above $75 \%$ consistently. Furthermore, this single animal belonged to the operated group. In consequence, testing was halted for all animals after 2000 trials and the mean percentage correct was used to compare groups. There was no significant difference $(t=-0.17$; df $4 ; p>0.05)$.

\section{Form discrimination}

Performance in nine-choice oddity where the correct stimulus differed from the other eight in either color and form or form alone was analyzed similarly. Savings scores for individual animals and means are presented in Figure 5.

Animals with V4 lesions showed a substantial impairment overall $(F=14.41$; df 1,$4 ; p<0.01)$. Both groups performed worse when the odd-one-out differed in form alone $(F=27.95$; df 1,$4 ; p<0.01)$. However, the saving scores show that animals with V4 lesions are impaired at selecting the odd-one-out whether it differs from the remaining items in both color and form or form alone $(F=0.38$; df 1,$4 ; p>0.05)$. The deficit in animals with V4 lesions in selecting the correct item in the former condition is a result of the substantial postoperative improvement

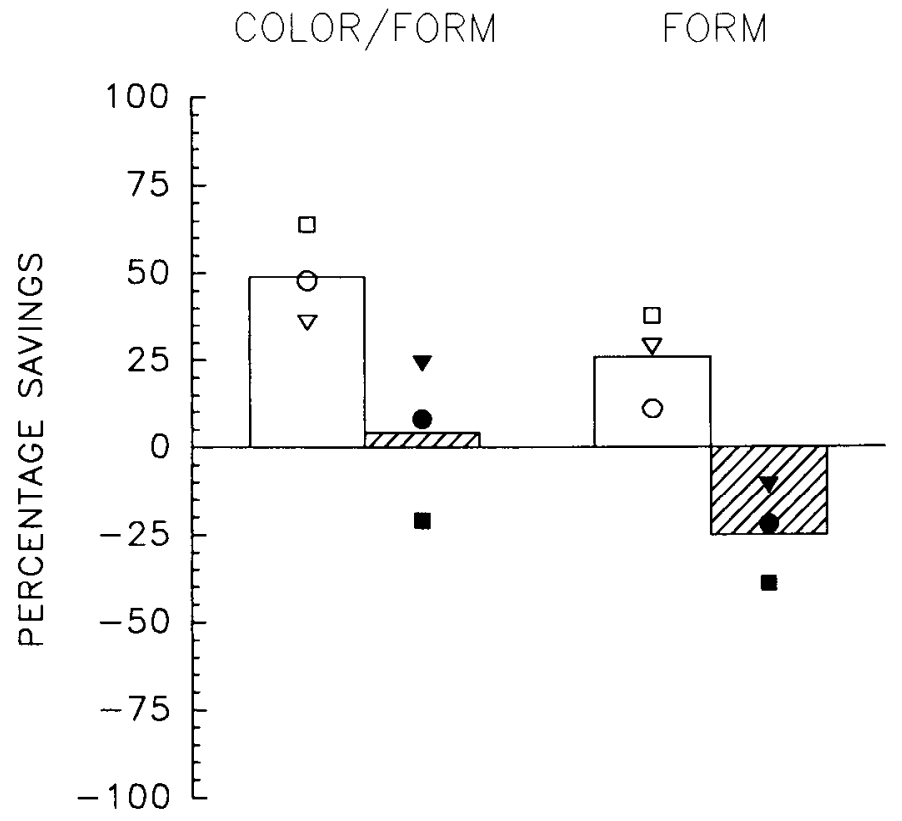

Figure 5. Saving scores for nine-choice oddity where the odd-one-out is distinguished by both color and form or by form alone. Scores for the unoperated control group are shown by open bars and for animals with V4 lesions by hatched bars. As in Figure 4, scores for individual animals are presented as open and solid symbols for the unoperated and operated groups, respectively.

shown by the unoperated control group (mean pre- and postoperative percentage correct, 66.7 and 88.5 , respectively). In contrast, animals with V4 lesions failed to show such an improvement (mean pre- and postoperative percentage correct, 78.4 and 77.2, respectively). Since the postoperative scores of two of the three operated animals were within the range of the unoperated control group, it is reasonable to conclude that the operated animals were not substantially impaired at oddity when the correct choice was indicated by differences in color and form and that the difference in saving scores was a result of different preoperative levels of performance. In striking contrast, while unoperated control animals showed a similar postoperative improvement in oddity for form (mean pre- and postoperative percentage correct, 54.4 and 73.8 , respectively), animals with V4 lesions showed a substantial decrement in performance (mcan pre- and postoperative percentage correct, 63.3 and 39.4, respectively). Taken together, these results indicate that V4 lesions impair form discrimination, with little effect on perception of color.

\section{Color discrimination}

Analysis of nine-choice color and gray discrimination was carried out using ANOVAs. Percentage correct performance as a function of difficulty of the discrimination is plotted for each of the color and gray oddity tasks in Figures 6 and 7, respectively. Animals with V4 lesions were indistinguishable from unoperated controls in their ability to perform color or gray oddity $(F$ $=0.25$, df $1,4, p>0.05 ; F=0.66$, df $1,4, p>0.05$, for color and gray oddity, respectively). In each case, animals performed better than would be expected on the basis of random responding for the most difficult discrimination and performance improved as differences in chromaticity and luminance increased $(F=49.47$, df $7,28, p<0.05 ; F=16.90$, df $5,20, p<0.05$, for color and gray oddity, respectively). This improvement was 


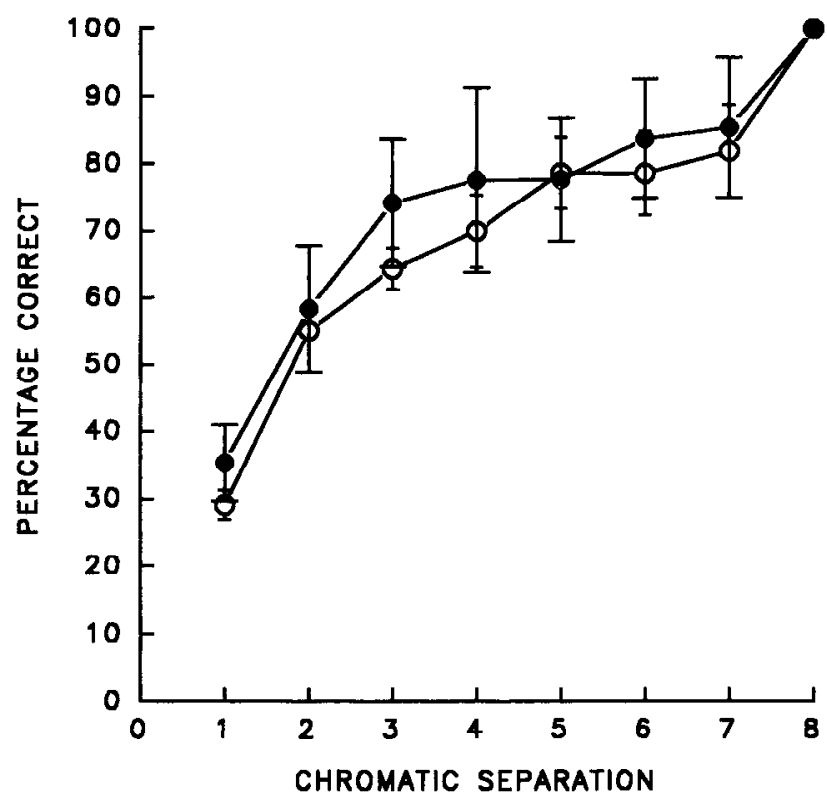

Figure 6. Percentage correct performance for nine-choice color oddity plotted against the chromatic separation of the stimuli. Separation refers to the distance apart of the target and nontarget items in a series of available discriminanda ordered in chromaticity from red to green. Error bars represent standard errors for the performance of the unoperated control group (open symbols) and animals with V4 lesions (solid symbols).

identical for each group $(F=0.41$, df $7,28, p<0.05 ; F=1.65$, df $5,20, p<0.05$, for color and gray oddity, respectively).

The two groups were indistinguishable in their ability to select the colored target from an array of grays that differed in luminance from one another. Mean scores for animals with V4 lesions and unoperated controls were $82.7 \%$ and $77.4 \%$, respectively $(t=1.13$; df $4 ; p>0.05)$. Similarly, they did not differ

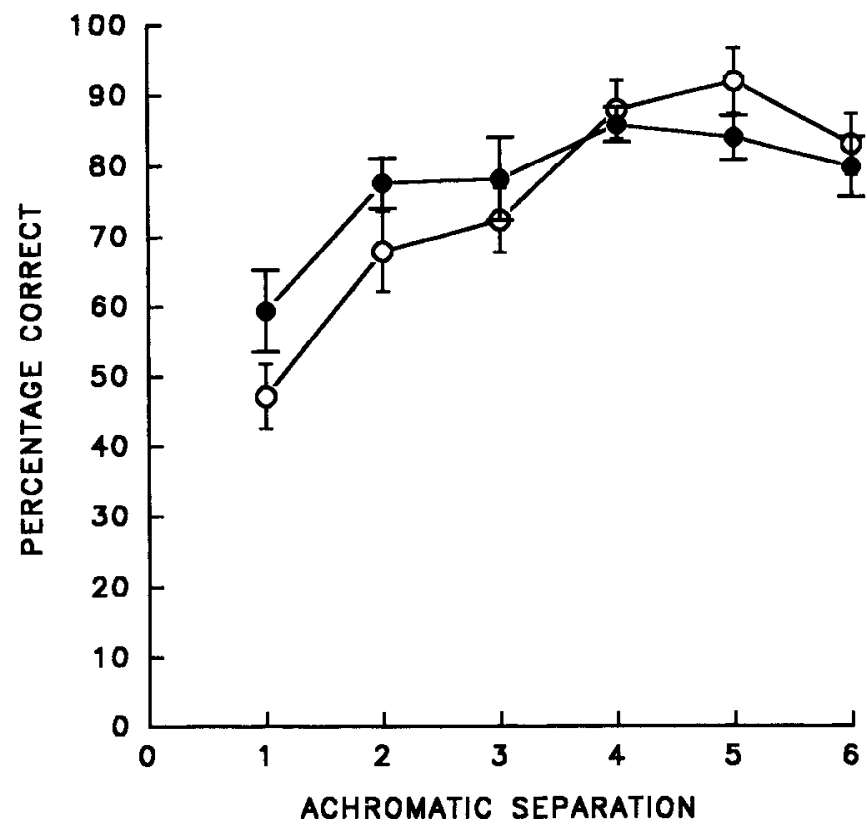

Figure 7. Mean percentage correct performance for each group plotted against task difficulty for nine-choice gray discrimination where separation refers to increasing luminance differences between the target and nontarget items. Error bars represent SE values for the performance of the unoperated control group (open symbols) and animals with V4 lesions (solid symbols).

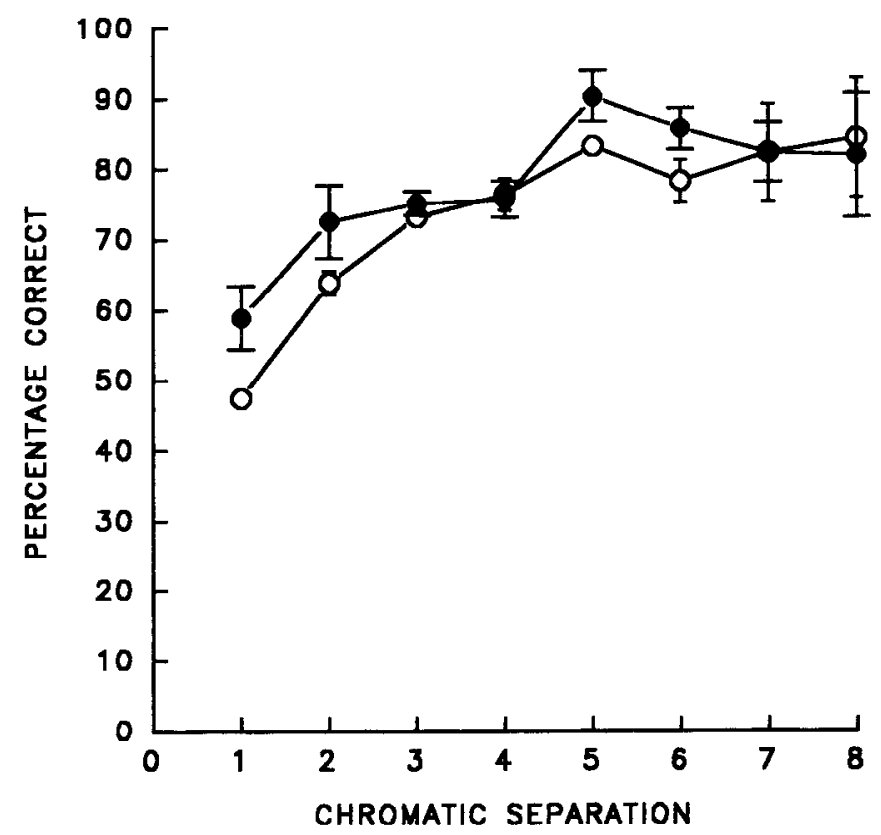

Figure 8. Percentage correct performance for three-choice color oddity plotted against the difficulty of the discrimination for the unoperated control group (open symbols) and animals with V4 lesions (solid symbols). Additional details are as in Figure 6.

in their ability to select the red patch from an array of green patches differing in luminance from one another. Mean scores for animals with V4 lesions and unoperated controls were $88.0 \%$ and $84.3 \%$, respectively $(t=0.68$; df $4 ; p>0.05)$.

The results of three-choice oddity discrimination were essentially identical to those for nine-choice discrimination. The performance of each group for color and gray discriminations is presented in Figures 8 and 9.

Again, there were no group differences in either overall performance at each discrimination $(F=0.78$, df $1,4, p<0.05 ; F$

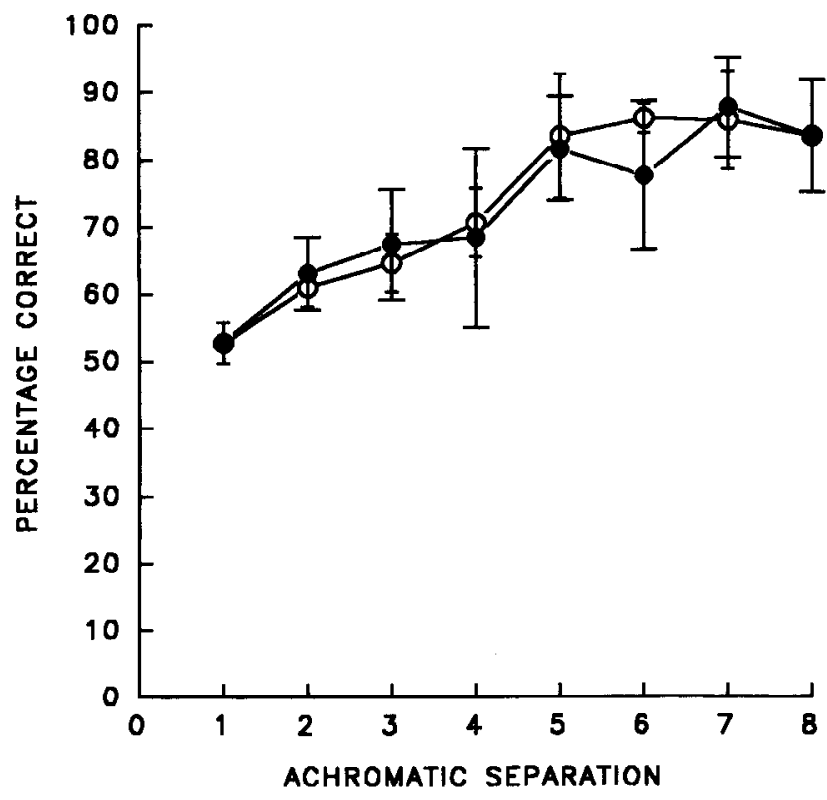

Figure 9. Percentage correct performance for three-choice gray oddity plotted against the difficulty of the discrimination for the unoperated control group (open symbols) and animals with V4 lesions (solid symbols). Additional details are as in Figure 6. 
$=0.00$, df 1,$4 ;, p<0.05$, for color and gray oddity, respectively) or in the improvement at easier discriminations $(F=1.25$, df $7,28, p<0.05 ; F=0.29$, df 7,28, $p<0.05$, for color and gray oddity, respectively). However, performance was no better than at chance levels for the most difficult discriminations (a stimulus separation of 1 unit in Figs. 8 and 9), demonstrating that the tasks were sufficiently difficult to reveal even minor impairments.

\section{Two-choice hue discrimination}

Mean errors to reach a criterion of $90 \%$ correct for the three tasks were $27.2,81.3$, and 4.7 for the unoperated animals, compared with $30.3,54.7$, and 3.67 for operated animals. Error scores did not differ between groups for any of the three twochoice discriminations $(t=-0.76$, df $4, p>0.05 ; t=0.79$, df $4, p>0.05 ; t=0.28$, $\mathrm{df} 4, p>0.05$, for the three discriminations, respectively.

\section{Discussion}

Shape discrimination. Substantial impairments in two-choice pattern discrimination following $\mathrm{V} 4$ ablation have been previously reported (Heywood and Cowey, 1987), and the severity of the shape discrimination in nine-choice oddity for alphanumeric characters confirms the earlier findings. This result should come as no surprise since cells tuned to orientation are common in V4 (Desimone and Schein, 1987) and the projections from area V2 to V4 suggest that information concerning shape as well as color must reach V4. In addition, area V4 provides the most substantial projection to inferotemporal cortex where cells respond to many attributes other than color (Tanaka et al., 1991). This also suggests a role for V4 in conveying much more than chromatic information.

The view that achromatopsia in humans is the result of damage to the homolog of area V4 in the monkey (Zeki, 1990) understandably relies heavily on the physiological properties of cells in area V4 and the connectivity of cortical visual areas in the monkey, yet an impairment in shape discrimination is not an invariable concomitant of achromatopsia and is certainly not its most severe characteristic. It has been suggested that in the absence of the form component of the $P$ channel, achromatopsic patients use the form system derived from the M channel (Zeki, 1990). This system, however, is clearly unable to mediate the discrimination of alphanumeric characters in monkeys that lack V4. The results from shape discrimination do not, therefore, support the view that it is distraction of area V4 per se in the human brain that causes achromatopsia, and this latter view is also queried by the evidence from color discrimination following $\mathrm{V} 4$ ablation in the monkey.

Color discrimination. The results of nine- and three-choice oddity discrimination for colors or grays did not reveal a difference between animals with V4 lesions and unoperated controls. In addition, V4 lesions did not disrupt the ability to select either the colored patch from an array of grays or the red patch from an array of greens where the elements of each array all differed in luminance. Yet a patient with cerebral achromatopsia tested on identical tasks failed to score better than would be expected on the basis of random responding (Heywood et al., 1991). These results are clearly at odds with the view that monkeys with V4 ablation are like patients with cerebral achromatopsia.

We previously reported a mild impairment in the discrimination of Munsell color samples following V4 removal in the monkey (Heywood and Cowey, 1987), and it may be wondered why no deficit at all could be found in the present experiment. In our previous experiment, the deficit in the green/yellow range was of the order of 2.5 Munsell hue steps, that is, a twofold increase in the just-noticeable difference. For technical reasons, the colors in the present experiment were equivalent to a series differing by approximately 5 hue steps on the Munsell scalc; limitations on the linearity of the display monitor prevented finer gradations of chromaticity to be reliably presented while maintaining isoluminance. A small hue discrimination deficit may therefore have been undetectable. However, the present experiment was not designed to assess small discrimination impairments of the magnitude reported previously, but rather to assess whether the impairment reported earlier had been grossly underestimated because of brightness differences between Munsell swatches that were not isoluminant. Clearly it had not. In the nine-choice oddity paradigm used here, discriminanda were distributed in widespread locations on the display screen where their luminances, even when intended to be identical, will vary. Such unintended departures from isoluminance, or when brightness is deliberately made irrelevant in the solution of the task, make it impossible that such cues were used in order to solve the task. The proposal that brightness differences may contribute to the ability to discriminate Munsell swatches was not, however, ill founded. The performance of achromatopsic patients in a three-choice oddity task (Heywood et al., 1987, 1991) showed good agreement with that of monkeys with V4 lesions. Large differences in chromaticity could still be discriminated. However, a patient with cerebral achromatopsia was unable to discriminate even more widely separated chromaticities when the discriminanda were presented on a display monitor and appropriately matched for luminance (Heywood et al., 1991).

There is a further, and potentially important, reason why monkeys with V4 ablation may have been unimpaired at hue discrimination in the present experiment. The stimuli were luminous in dark surroundings, with negligible reflected light. As a result, their spectral composition was invariant, no matter what the position of the monkey. In our earlier experiment (Heywood and Cowey, 1987), the stimuli were Munsell hues, seen entirely by reflected light. However, the spectral composition of the reflected light seen by the animal must vary considerably. As the animal alters its position, the stimuli will reflect varying amounts of light directly incident from the source and indirectly from light scattered from the walls of the testing cubicle onto the stimuli. In other words, the animal would need color constancy in order to deal with the problem. If area V4 is selectively involved in color constancy as first suggested by Zeki (1983b), its removal might impair the discrimination of surface colors seen by reflected light, but not purely luminous colors.

Our task of color ordering more closely resembles the Farnsworth-Munsell 100-Hue Test, conventionally used in the assessment of color discrimination in achromatopsic patients, than discrimination in an oddity test. Monkeys with V4 lesions made more errors postoperatively than unoperated controls in reaching criterion on all versions of the task. More importantly, however, the V4 animals were just as impaired on the ordering of achromatic grays as they were on the ordering of the red/green series of discriminanda used in the oddity tasks. In other words, their impairment was not color specific. Ordering of the additional series of colors was more difficult for both groups but particularly so for the operated animals. Whether the more extended range of chromaticities or the more closely spaced hues in some of the series contributed to this difference is unclear; 
that is, a mild hue impairment in animals with V4 lesions may have made this task more difficult. However, the simplest explanation for the poor performance of operated animals on all of the ordering tasks is that their gross impairment in pattern discrimination resulted in difficulty in discriminating ordered from disordered arrays regardless of whether the stimuli were chromatic or achromatic. In this respect, monkeys with V4 lesions again differ markedly from patients with frank achromatopsia. One such patient, with accompanying apperceptive agnosia, had little difficulty on identical tasks of chromatic and achromatic ordering (Heywood et al., 1991). One likely explanation for this preserved ability is that cells in the broad-band $M$ channel, whether in the magnocellular layers of the dLGN or in area MT (V5) or areas V3 and V3A of the monkey, which do respond to isoluminant chromatic borders without coding the chromaticities that compose the border (Saito et al., 1989; Hubel and Livingstone, 1990), may allow such discriminations to be performed in the absence of any conscious experience of the hues themselves. For example, the chromatic borders between contiguous but very different hues presumably provoke brisker signals than the borders between similar hues. Disordered series of isoluminant hues are therefore likely to yield different border signals than spectrally ordered series of the same hues. Thus, patients may use their preserved pathways that are specialized for the processing of form to solve the chromatic ordering task, and questioning such a patient about his performance confirms this (Heywood et al., 1991). Again, monkeys with V4 lesions differ from the patient with achromatopsia in that they show the opposite pattern of deficits-preserved color discrimination ability with a substantial impairment in the discrimination of shape. However, the proposal that the achromatopsic patient has a selective loss of the $P$ channel and is solely reliant on the $\mathrm{M}$ channel is unlikely. The spectral sensitivity function of one achromatopsic patient (Heywood et al., 1991) was normal in form and showed evidence for opponent processing, a property that is confined to the $\mathrm{P}$ channel, and input from the short-wavelength cones, which provide little or no input to the M channel (DeValois and Jacobs, 1968; Gouras, 1968; King-Smith and Carden, 1976; Derrington et al., 1984; Lec and Strohmeycr, 1989).

Nevertheless, a reliance of normal animals on the chromatic borders between adjacent patches of color in the solution of ordering tasks was apparent. When a small achromatic strip was placed between each patch, performance of all animals was disrupted but not more so for operated animals. Again, these results do not support a simple correspondence between area V4 in the monkey and the area implicated in human achromatopsia. Introducing a narrow strip between each patch resulted in chance performance in an achromatopsic patient for the ordering of chromatic, but not achromatic, stimuli (Heywood et al., 1991).

In conclusion, the weight of evidence is against area V4 as a candidate for the cortical registration of color in macaque monkeys and that it is indispensable to normal color discrimination. Furthermore, it is not clear that the human homolog of V4 is the "color center" located in the lingual and fusiform gyri, destruction of which results in the profound loss of color sensation in the clinical condition of achromatopsia.

However, the proposed homology could be preserved by supposing that achromatopsia requires destruction of this area of cortex together with the underlying white matter, which can be minimally damaged by subpial ablation in monkeys. This supposition would be supported by the existence of patients with damage to the fusiform gyrus that spared the white matter and did not produce achromatopsia. Unfortunately, such "negative" findings are rarely if ever reported.

\section{References}

Damasio A, Yamada T, Damasio H, Corbett J, McKee J (1980) Central achromatopsia: behavioural, anatomic, and physiologic aspects. Neurology 30:1064-1071.

Derrington AM, Krauskopf J, Lennie P (1984) Chromatic mechanisms in lateral geniculate nucleus of macaque. J Physiol (Lond) 357:241265

Desimone R, Schein SJ (1987) Visual properties of neurons in area V4 of the macaque: sensitivity to stimulus form. J Neurophysiol 57: 835-868

DeValois RL, Jacobs GH (1968) Primate color vision. Science 162: 533-540.

Gallyas F (1969) Silver staining of myelin by means of physical development. Neurol Res 1:203-209.

Gouras P (1968) Identification of cone mechanisms in monkey ganglion cells. J Physiol (Lond) 199:533-547.

Heywood CA, Cowey A (1987) On the role of cortical area V4 in the discrimination of hue and pattern in macaque monkeys. J Neurosci 7:2601-2617.

Heywood CA, Wilson B, Cowey A (1987) A case study of cortical colour "blindness" with relatively intact achromatic discrimination. J Neurol Neurosurg Psychiatry 50:22-29.

Heywood CA, Cowey A, Newcombe F (1991) Chromatic discrimination in a cortically blind observer. Eur J Neurosci 8:802-812.

Hubel DH, Livingstone MS (1990) Color and contrast sensitivity in the lateral geniculate body and primary visual cortex of the macaque monkey. J Neurosci 10:2223-2237.

King-Smith PE, Carden D (1976)Luminance and opponent-color contributions to visual detection and adaptation and to temporal and spatial integration. J Opt Soc Am 66:709-717.

Land EH (1974) The retinex theory of color vision. Proc R Soc Gr $\mathrm{Br}$ 43:27-58.

Lee J, Strohmeyer CF (1989) Contributions of human short-wave cones to luminance and motion detection. J Physiol (Lond) 413:563593.

Livingstone MS, Hubel DH (1987) Segregation of form, color and stereopsis in primate area 18. J Neurosci 7:3378-3415.

Lueck CJ, Zeki S, Friston KJ, Deiber MP, Cope P, Cunningham VJ, Lammertsma AA, Kennard C, Frackowiak RS (1989) The colour centre in the cerebral cortex of man. Nature 340:386-389.

Maunsell JHK, Nealy TA, DePriest DD (1990) Magnocellular and parvocellular contributions to responses in the middle temporal visual area (MT) of the macaque monkey. J Neurosci 10:3323-3334.

Meadows JC (1974) Disturbed perception of colours associated with localized cerebral lesions. Brain 97:615-632.

Perry VH, Oehler R, Cowey A (1984) Retinal ganglion cells that project to the dorsal lateral geniculate nucleus in the macaque monkey. Neuroscience 12:1101-1123.

Saito H, Tanaka K, Isono H, Yasuda M, Mikami A (1989) Directionally selective response of cells in the middle temporal area (MT) of the macaque monkey to the movement of equiluminous opponent colour stimuli. Exp Brain Res 75:1-14.

Schiller PH, Lee K (1991) The role of the primate extrastriate area $\mathrm{V} 4$ in vision. Science 251:1251-1253.

Schiller PH, Logothetis NK (1990) The color-opponent and broadband channels of the primate visual system. Trends Neurosci 13:392398.

Tanaka K, Saito HA, Fukuda Y, Moriya M (1991) Coding visual images of objects in the inferotemporal cortex of the macaque monkey. J Neurophysiol 66:170-189.

Ungerleider LG (1985) The corticocortical pathways for object recognition and spatial perception. In: Pattern recognition mechanisms (Chagas C, Gattass R, Gross CG, eds), pp 21-37. Vatican City: Pontifical Academy of Sciences.

Zeki SM (1983) Colour coding in the cerebral cortex: the reaction of cells in monkey visual cortex to wavelengths and colours. Neuroscience 9:741-765.

Zeki SM (1990) A century of cerebral achromatopsia. Brain 113:17211777 